\title{
The bound state corrections to the semileptonic decays of the heavy baryons
}

\author{
I. D'Souza ${ }^{(a)}$, C. S. Kalman ${ }^{(a)}$, P. Yu Kulikov ${ }^{(b)}$ and I. M. Narodetskii ${ }^{(b)}$ \\ ${ }^{(a)}$ Concordia University, Montreal, Canada \\ ${ }^{(b)}$ Institute of Theoretical and Experimental Physics, Moscow, RF
}

We present an investigation of the lepton energy distributions in the inclusive semileptonic weak decays of heavy baryons performed within a relativistic quark model formulated on the light front (LF). Using the heavy-quark LF distribution functions related to the equal time momentum wave functions taken from the Kalman-Tran-D'Souza model we compute the electron energy spectra and the total semileptonic widths of the $\Lambda_{Q}$ and $\Xi_{Q}(Q=c, b)$ baryons and confront the results with existing data.

\section{Introduction}

It is important to study the differential distributions in semileptonic decays of heavy flavors in order to extract the CKM matrix elements $\left|V_{c b}\right|$ and $\left|V_{u b}\right|$, key phenomenological parameters of the Standard Model. To this end one needs to disentagle the effects of the strong interactions at large distances from the quark-gluon Lagrangian known at small distances. Since the $b$-quark is heavy compared to the QCD scale, the inclusive semileptonic decays can be treated with the help of an operator product expansion (OPE) combined with the heavy quark expansion (HQE) 1]. The result (away from the endpoint of the spectrum) is that the inclusive differential decay width $d \Gamma / d E_{\ell}$ ( $E_{\ell}$ is the lepton energy) may be expanded in $\Lambda / m_{b}$, where $\Lambda$ is a QCD related scale of order $500 \mathrm{MeV}$, and $m_{b}$ is the mass of the heavy quark. The leading term (zeroth order in $\Lambda / m_{b}$ ) is the free quark decay spectrum, the subleading term vanishes, and the subsubleading term involves parameters from the heavy quark theory, but should be rather small, as it is of order $\left(\Lambda / m_{b}\right)^{2}$. However, near the end point the $1 / m_{b}$ expansion has to be replaced by an expansion in twist. To describe this region one has to introduce a so-called "shape function", which in principle introduces a large hadronic uncertainty. Since a model independent determination of the shape function is not available at the present time, a certain model dependence in this region seems to be unavoidable.

The global prediction of OPE is that the lepton spectra from the semileptonic decays of the heavy flavor hadrons are all alike with accuracy up to $\mathcal{O}\left(1 / m_{b}^{3}\right)$ except for the end point region. However, it is well known that the similar OPE prediction for the $\Lambda_{b}$ lifetime is puzzling because it predicts a value of $\tau_{B} / \tau_{\Lambda_{B}}$, which is considerably smaller than determined experimentally. It is not clear whether the present contradiction between the theory and the data is a temporary difficulty, or whether it implies a fundamental flaw in the OPE approach. In this respect, the use of phenomenological models, like the constituent quark model, could be of great interest as a complementary approach to the $O P E$ method.

\section{The light-front model}

Two phenomenological approaches had been applied to describe strong interaction effects in the inclusive weak decays: the parton ACM model amended to include the motion of the heavy quark inside the decaying hadron [2], and the "exclusive model" based on the summation of different channels, one by one [3]. The various light-front (LF) approaches to consideration of the inclusive semileptonic transitions were suggested in Refs. [4]- [6]. Each of them needs an input from constituent quark model to parametrize non-perturbative effects.

In this paper, we evaluate the nonperturbative corrections to the lepton spectrum in the inclusive semileptonic decays of heavy flavor baryons using an approach of Ref. [6] in which non-perturbative $Q C D$ effects in a heavy baryon are mocked up by a constituent quark model wave function $\psi\left(\xi, p_{\perp}^{2}\right)$ on the $\mathrm{LF} .\left|\psi\left(\xi, p_{\perp}^{2}\right)\right|^{2}$ represents the probability to find the $Q$ quark carrying a LF fraction $\xi$ and a transverse momentum squared $p_{\perp}^{2}=$ $\left|\mathbf{p}_{\perp}\right|^{2}$. The calculations of the inclusive semileptonic widths and the corresponding branching ratios found here introduce no new parameters. It is based solely upon the eigenfunctions for baryon states found by Kalman, Tran and D'Souza (see [7]). Our main purpose here is to confront the lepton spectra from semileptonic decays of the different baryons.

${ }^{1}$ Here and below the symbol $Q=b, c$ denotes the heavy quark
and the symbol $H_{Q}$ denotes the heavy hadron. 
The general expression for the distribution over lepton energy is based on the standard Lorentzinvariant kinematical analysis (see e.g. [8]):

$$
\begin{aligned}
\frac{d \Gamma_{S L}}{d y}= & \frac{G_{F}^{2} m_{H_{Q}}^{5}}{64 \pi^{3}}\left|V_{Q^{\prime} Q}\right|^{2} \int_{0}^{t_{\max }} d t \int_{s_{0}}^{s_{\max }} d s \\
& \left\{\begin{array}{c}
t W_{1}+\frac{1}{2}\left[y(1+t-s)-y^{2}-t\right] W_{2} \\
+t\left[\frac{1+t-s}{2}-y\right] W_{3}+\ldots
\end{array}\right\}
\end{aligned}
$$

where $y=2 E_{\ell} / m_{H_{Q}}, t=q^{2} / m_{H_{Q}}^{2}, s=M_{X_{Q^{\prime}}}^{2} / m_{H_{Q}}^{2}$, $q=p_{\ell}+p_{\nu_{\ell}}, M_{X_{Q}^{\prime}}^{2}=\left(p_{H_{Q}}-q\right)^{2}$ and the structure functions $W_{i}=W_{i}(s, t)$ appear in the decomposition of the hadronic tensor $W_{\alpha \beta}$ in Lorentz covariants. The ellipsis in (2) denotes the terms proportional to the lepton mass squared. The kinematical limits of integration can be found from equation

$\frac{s}{1-y}+\frac{t}{y} \leq 1$

They are given by $0 \leq y \leq y_{\max }, s_{\max }=1+t-$ $(y+t / y)$, and $t_{\max }=y\left(\left(1-\left(1-y_{\max }\right) /(1-y)\right)\right.$, with $y_{\max }=1-\left(m_{X_{Q^{\prime}}^{(m i n)}} / m_{H_{Q}}\right)^{2}$ and $m_{X_{Q^{\prime}}}^{(\min )}$ being the minimal mass of the hadronic state $X_{Q^{\prime}}$.

In a parton model of Ref. [6] inclusive semileptonic $H_{Q} \rightarrow X_{Q}^{\prime} \ell \nu_{\ell}$ decay is treated in a direct analogy to deep-inelastic scattering. Specifically, it is assumed that the sum over all possible final states $X_{Q^{\prime}}$ can be modelled by the decay width of an onshell $Q$ quark into on-shell $Q^{\prime}$-quark weighted with the $Q$-quark distribution $\left|\psi\left(\xi, p_{\perp}^{2}\right)\right|^{2}$. The natural variables for $\psi\left(\xi, p_{\perp}^{2}\right)$ are the light-cone momentum fraction $\xi=p_{Q}^{+} /\left(p_{1}^{+}+p_{2}^{+}+p_{Q}^{+}\right)$and the $Q$ quark transverse momentum $p_{\perp}^{2}=\mathbf{p}_{Q \perp}$. The function $\left|\psi\left(\xi, p_{\perp}^{2}\right)\right|^{2}$ has support $0 \leq \xi \leq 1$ and $0 \leq p_{\perp}^{2} \leq \infty$. This function as a function of $\xi$ has the maximum at $\xi=m_{Q} /\left(m_{Q}+m_{1}+m_{2}\right)$, at smaller values of $\xi$ it rapidly vanishes. The normalization condition reads

$\pi \int_{0}^{1} d \xi \int d p_{\perp}^{2}\left|\psi\left(\xi, p_{\perp}^{2}\right)\right|^{2}=1$.

Following the above assumption, the hadronic tensor $W_{\alpha \beta}$ is written as

$$
\begin{aligned}
W_{\alpha \beta}= & \int w_{\alpha \beta}^{\left(Q^{\prime} Q\right)}\left(p_{Q^{\prime}}, p_{Q}\right) \delta\left[\left(p_{Q}-q\right)^{2}-m_{Q^{\prime}}^{2}\right] \\
& \frac{\left|\psi\left(\xi, p_{\perp}^{2}\right)\right|^{2}}{\xi} \theta\left(\varepsilon_{Q^{\prime}}\right) d \xi d^{2} p_{\perp},
\end{aligned}
$$

where

$w_{\alpha \beta}^{\left(Q^{\prime} Q\right)}\left(p_{Q^{\prime}}, p_{Q}\right)=\frac{1}{2} \sum_{\text {spins }} \bar{u}_{Q^{\prime}} O_{\alpha} u_{Q} \cdot \bar{u}_{Q} O_{\beta}^{+} u_{Q^{\prime}}$,

with $O_{\alpha}=\gamma_{\alpha}\left(1-\gamma_{5}\right)$. The factor $1 / \xi$ in Eq. (四) is due to the normalization of the hadron-quark vertex [9]. The hadronic tensor (4) differs from the corresponding expressions of Refs. [4 and [5] by the non-trivial dependence on $p_{\perp}^{2}$ which enters both $\left|\psi\left(\xi, p_{\perp}^{2}\right)\right|^{2}$ and argument of the $\delta$-function. The calculation of the structure functions $W_{i}(t, s)$ from Eq. (1) is straightforward. For details see Appendix of [6].

Note that in the heavy-quark limit, $m_{Q} \rightarrow$ $\infty$, the $Q$-quark distribution in a heavy hadron becomes a delta function peaked at $\xi=1$, which implies that the semileptonic width of a heavy hadrons coincides with that of a heavy quark. At finite values of the $Q$-quark mass the result for $\Gamma_{S L}$ exhibits in general an $m_{Q}$-dependence of the following general form: $\Gamma_{S L} \propto m_{Q}^{5}\left[1+c / m_{Q}+O\left(1 / m_{Q}^{2}\right)\right]$, where $c$ is a non-vanishing coefficient depending on the particular quark model adopted. However, the mass $m_{Q}$ is the constituent mass of the $b$-quark, which may differ from the pole quark mass $m_{Q}^{\text {pole }}$ commonly appearing in the $O P E$ of the heavy hadron decay rate (see below). Assuming $m_{Q}=m_{Q}^{\text {pole }}-c / 5+O\left(1 / m_{Q}^{\text {pole }}\right)$, the well-known result [1] of the absence of the $1 / \mu_{Q}$ corrections to the free-quark decay may be recovered. The above argument is completely analogous to that used to eliminate the $1 / m_{b}$ corrections from the total width in the ACM model [10].

\section{The electron spectrum from semileptonic B decays}

Before calculating the electron spectra for heavy baryons we illustrate our approach with an example of the electron spectrum from the inclusive semileptonic B decays [11].

Since we do not have an explicit representation for the B-meson Fock expansion in QCD, we proceed by making an ansätz for $\psi\left(\xi, p_{\perp}^{2}\right)$. This is a model dependent enterprise but has its close equivalent in studies of electron spectra using the ACM model. We first make an ansätze for the momentum space structure of an equal time (ET) quark model wave function $\Phi\left(\mathbf{p}^{2}\right)$ and then convert from ET to LF momenta by leaving the transverse momenta unchanged and letting

$p_{i z}=\frac{1}{2}\left(p_{i}^{+}-p_{i}^{-}\right)=\frac{1}{2}\left(p_{i}^{+}-\frac{p_{i \perp}^{2}+m_{i}^{2}}{p_{i}^{+}}\right)$

for both the $b$-quark $(i=b)$ and the quark-spectator 
$(i=s p)$. Because the ET function depends on the relative momentum it is more convenient to use the quark-antiquark rest frame instead of the $\mathcal{B}$-meson rest frame. Recall that in the LF formalism these two frames are different. Then the longitudinal LF momentum fractions $\xi_{i}$ are defined as $\xi_{s p}=p_{s p}^{+} / M_{0}$, $\xi_{b}=p_{b}^{+} / M_{0}$, where the free mass operator $M_{0}^{2}$ is defined as

$$
\begin{aligned}
M_{0}^{2}= & \left(\sqrt{m_{Q}^{2}+\mathbf{p}^{2}}+\sqrt{m_{s p}^{2}+\mathbf{p}^{2}}\right)^{2}= \\
= & \frac{p_{\perp}^{2}+m_{b}^{2}}{\xi}+\frac{p_{\perp}^{2}+m_{s p}^{2}}{1-\xi},
\end{aligned}
$$

with $\mathbf{p}^{2}=p_{\perp}^{2}+p_{z}^{2}$, and

$p_{z}=\left(x-\frac{1}{2}\right) M_{0}-\frac{m_{b}^{2}-m_{s p}^{2}}{2 M_{0}}$.

The distribution function $\left|\psi\left(\xi, p_{\perp}^{2}\right)\right|^{2}\left(\xi=\xi_{b}\right)$ normalized according to (3) is given by $\left|\psi\left(\xi, p_{\perp}^{2}\right)\right|^{2}=$ $\left|\partial p_{z} / \partial \xi\right|^{2} \Phi\left(p_{\perp}^{2}+p_{z}^{2}\left(\xi, p_{\perp}^{2}\right)\right)$. Explicit form of $\left|\partial p_{z} / \partial \xi\right|$ is given e.g. by Eq. (22) of Ref. [6]. In this calculation the equal time momentum distribution $\Phi\left(\mathbf{p}^{2}\right)$ of a quark-spectator is taken in the standard Gaussian form

$\Phi\left(\mathbf{p}^{2}\right)=\frac{4}{\sqrt{\pi} p_{F}^{3}} \exp \left(-\frac{\mathbf{p}^{2}}{p_{F}^{2}}\right)$,

with the Fermi momentum $p_{F}=0.4 \mathrm{GeV}$. The calculation uses $m_{b}=4.8 \mathrm{GeV}$ (the average value of the floating $b$-quark mass in the ACM model) and $m_{c}=1.5 \mathrm{GeV}$.

We have implicitly included the $\mathcal{O}\left(\alpha_{s}\right)$ perturbative corrections with $\alpha_{s}=0.25$ arising from gluon Bremsstrahllung and one-loop effects which modify an electron energy spectra at the partonic level. It is customary to define a correction function $G(x)$ to the electron spectrum $d \Gamma_{b}^{(0)}$ calculated in the tree approximation for the free quark decay through

$\frac{d \Gamma_{b}}{d x}=\frac{d \Gamma_{b}^{(0)}}{d x}\left(1-\frac{2 \alpha_{s}}{3 \pi} G(x)\right)$,

where $x=2 E / m_{b}$. In actual calculations we neglect the terms $\sim m_{c}^{2} / m_{b}^{2}$ in $G(x)$ and take this function from 13 .

We display in Fig. 1 the results of our calculations of the electron spectrum in the inclusive semileptonic $\mathcal{B}$ decays. We find an overall agreement with the published experimental data from the CLEO collaboration 12]. A more detailed fit to the measured spectrum can impose constraints on the distribution function and the mass of the charm

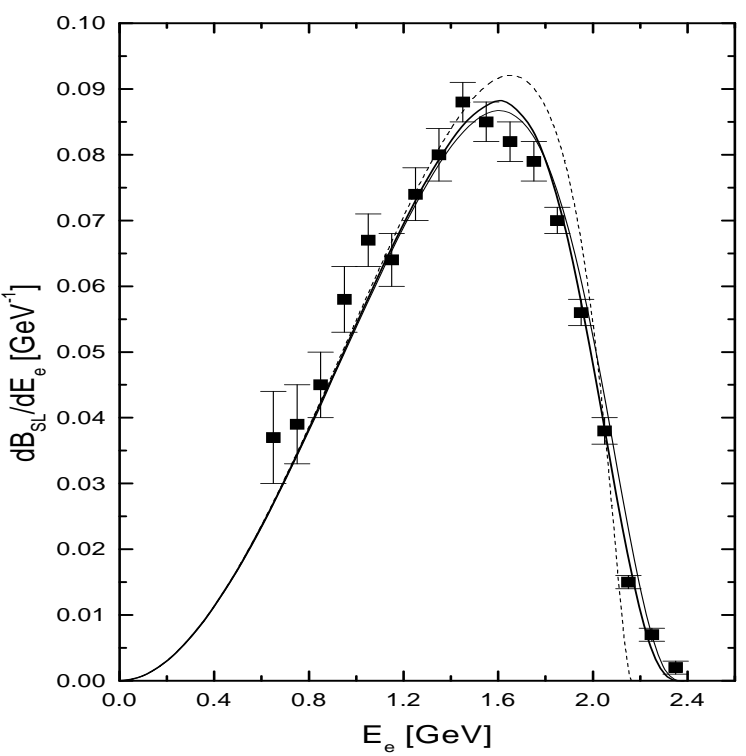

Figure 1. The predicted electron energy spectrum the semileptonic $\mathcal{B}$ decays compared with the CLEO data 12]. Thick solid line is the LF result, thin solid line is the ACM result, dashed line refers to the free quark decay.

quark. Such the fit should also account for detector resolution. The LF and ACM spectra are normalized to $10.71 \%, 10.84 \%$, respectively. $\left|V_{c b}\right|=0.042$. Using $\tau_{B}=1.653 \pm 0.028 \mathrm{ps}^{-1}$ 14 one obtains $\Gamma\left(\mathcal{B} \rightarrow X_{c} e \bar{\nu}\right)=(6.51 \pm 0.11) \times 10^{-2} p s^{-1} \quad(\mathrm{LF})$, and $\Gamma\left(\mathcal{B} \rightarrow X_{c} e \bar{\nu}\right)=(6.56 \pm 0.11) \times 10^{-2} p s^{-1}(\mathrm{ACM})$, that agrees within the error bars with the experimental result $\Gamma_{\exp }\left(b \rightarrow X_{c} e \bar{\nu}\right)=(6.67 \pm 0.2) \times 10^{-2} \mathrm{ps}^{-1}$ (for $\left.\left|V_{c b}\right|_{\text {incl }}=(40.4 \pm 1.07) \times 10^{-3}\right)$, quoted in the forthcoming PDG2002 [14] 2.

\section{The electron spectrum for the heavy baryon semileptonic decays}

We proceed to present numerical results for the lepton spectra in the heavy baryon decays. We consider the problem of a constituent bound state formed by a heavy quark interacting with the lighter ones. The spectator quarks have a momentum distribution $\Phi\left(\mathbf{p}_{1}, \mathbf{p}_{2}\right)$ where $\mathbf{p}_{1}$ and $\mathbf{p}_{2}$ are their 3momenta. Specifically, in the Kalman-Tran-D'Souza model in the first approximation the momentum dis-

\footnotetext{
${ }^{2}$ It should be noted that $\Gamma_{\text {exp }}$ in 14 involves a weighted average of the $\mathcal{B}$ mesons and $\Lambda_{b}$ baryons produced in $Z^{0}$ decays (hence the symbol $b$ ) different from the corresponding one given by CLEO, which has been measured at the $\Upsilon(4 S)$ resonance.
} 
tribution of spectator quarks is:

$$
\begin{aligned}
\Phi\left(\mathbf{p}_{1}, \mathbf{p}_{2}\right)= & \left(\frac{1}{\alpha \sqrt{\pi}}\right)^{3}\left(\frac{1}{\beta \sqrt{\pi}}\right)^{3} \\
& \times \exp \left(-\frac{\mathbf{k}^{2}}{\alpha^{2}}\right) \exp \left(-\frac{\mathbf{p}_{Q}^{2}}{\beta^{2}}\right),
\end{aligned}
$$

where $\mathbf{k}=\left(m_{2} \mathbf{p}_{1}-m_{1} \mathbf{p}_{2}\right) /\left(m_{1}+m_{2}\right), m_{1}$ and $m_{2}$ being the masses of the spectator quarks. The parameters $\alpha$ and $\beta$ were are obtained from the fit to the spectra in Refs. [7]. These parameters are listed in Table 2 of Ref. 15 where the comparison of the theoretical predictions of the Kalman-D'Souza model for the heavy baryon masses with the experimental data was also given. The heavy quark distribution function is given by

$$
\begin{aligned}
F\left(\xi, \mathbf{p}_{Q \perp}\right)= & \frac{1}{8} \int_{0}^{1-\xi} d \eta \int d \mathbf{k}_{\perp} \Phi\left(\mathbf{p}_{1}, \mathbf{p}_{2}\right) \\
& \times\left(M_{0}+\frac{e_{Q}}{\xi M_{0}}\right)\left(M_{0}+\frac{e_{1}}{\eta M_{0}}\right) \\
& \times\left(M_{0}+\frac{e_{2}}{(1-\xi-\eta) M_{0}}\right)
\end{aligned}
$$

where $\eta=p_{1}^{+} / M_{0}, e_{Q}=\left(p_{Q \perp}^{2}+m_{Q}^{2}\right) / \xi, e_{1}=\left(k_{\perp}^{2}+\right.$ $\left.m_{1}^{2}\right) / \eta, e_{2}=\left(\left(\mathbf{k}_{\perp}-\mathbf{p}_{Q \perp}\right)^{2}+m_{2}^{2}\right) /(1-\xi-\eta)$, and the free mass operator $M_{0}^{2}$ is now given by $M_{0}^{2}=$ $e_{Q}+e_{1}+e_{2}$. In terms of the $L F$ variables $\mathbf{k}^{2}$ and $\mathbf{p}_{Q}^{2}$ are given by

$$
\begin{aligned}
\mathbf{k}^{2}= & \mathbf{k}_{\perp}^{2}-\frac{2 m_{1}}{m_{1}+m_{2}} \mathbf{k}_{\perp} \mathbf{p}_{Q \perp}+\left(\frac{m_{1}}{m_{1}+m_{2}}\right)^{2} \mathbf{p}_{Q \perp}^{2} \\
& +\frac{M_{0}^{2}}{4} \times\left(\eta+\frac{m_{1}}{m_{1}+m_{2}} \xi\right. \\
& \left.-\frac{m_{1}}{m_{1}+m_{2}} \cdot \frac{e_{Q}}{M_{0}^{2}}-\frac{e_{1}}{M_{0}^{2}}\right)^{2}
\end{aligned}
$$

and

$\mathbf{p}_{Q}^{2}=\mathbf{p}_{Q \perp}^{2}+\frac{1}{4}\left(\xi M_{0}-\frac{e_{Q}}{M_{0}}\right)^{2}$.

In the calculation of the lepton spectra instead of the identification $m_{Q}^{L F}=<m_{f}^{A C M}>$ used in the $\mathcal{B}$ meson case before we use the original values of the quark masses [7]

$$
\begin{aligned}
& m_{u}=m_{d}=0.23 \mathrm{GeV}, \quad m_{s}=0.605 \mathrm{GeV} \\
& m_{c}=1.961 \mathrm{GeV}, \quad m_{b}=5.637 \mathrm{GeV}
\end{aligned}
$$

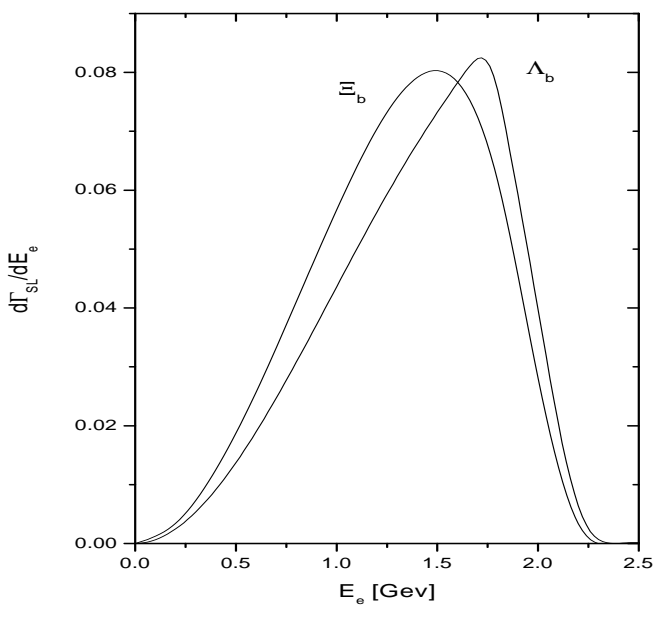

Figure 2. The predicted electron energy spectrum for $\Lambda_{b}$ and $\Xi_{b}$ semileptonic decays. The spectra normalized to $\Gamma_{S L}\left(\Lambda_{b}\right)=0.086 \mathrm{ps}^{-1}$ and $\Gamma_{S L}\left(\Xi_{b}\right)=0.092$ $\mathrm{ps}^{-1} \cdot\left|V_{c b}\right|=0.04$

obtained from the fit to heavy baryon spectra. Note that the large masses of the heavy constituent quarks in the model of Ref. [7] introduce huge factors $\left(m_{b} / m_{b, \text { pole }}\right)^{5} \sim 2.2$ and $\left(m_{c} / m_{c, \text { pole }}\right)^{5} \sim 3.8 \mathrm{in}$ the semileptonic rate of the heavy b- and c-hadrons. These factors however are cancelled to the great extent by the bound state factor in Eq. (4).

We display the electron spectra for the inclusive semileptonic decays $\Lambda_{b}$ and $\Xi_{b}$ in Fig. 2 and for the inclusive semileptonic decays $\Lambda_{c}$ and $\Xi_{c}$ in Fig. 3. The calculation uses the LF distribution function (12) of the heavy quark derived from the ET D'Souza-Kalman momentum distribution (11). The perturbative $\mathcal{O}\left(\alpha_{s}\right)$ corrections are also included.

The total inclusive semileptonic width $\Gamma\left(\Lambda_{b} \rightarrow X_{c} e \nu_{e}\right)=0.086 \cdot\left|V_{c b} / 0.04\right|^{2} \mathrm{ps}^{-1}$ obtained by a direct integration of the spectrum coincides with that found in Ref. [15]. This rate, though a bit large, agrees with the estimation based on the semiinclusive branching ratio which (using $\left.\tau_{\Lambda_{b}}=1.229 \pm 0.080 \mathrm{ps}^{-1}\right)$ implies $\Gamma\left(\Lambda_{b} \rightarrow\right.$ $\left.X_{c} e \nu_{e}\right)=\left(7.97_{-3.09}^{+2.52}\right) \times 10^{-2} p s^{-1}$. The predicted exclusive/inclusive semileptonic ratio (based on the estimation $\Gamma\left(\Lambda_{b} \rightarrow \Lambda_{c}\right)=0.052 \mathrm{ps}^{-1}$ [17) is

$R_{E}\left(\Lambda_{b}\right)=\frac{\Gamma\left(\Lambda_{b} \rightarrow \Lambda_{c} e \nu\right)}{\Gamma\left(\Lambda_{b} \rightarrow X_{c} e \nu\right)}=57 \%$,

close to the meson value $R_{E}(\mathcal{B})=66 \%$. 


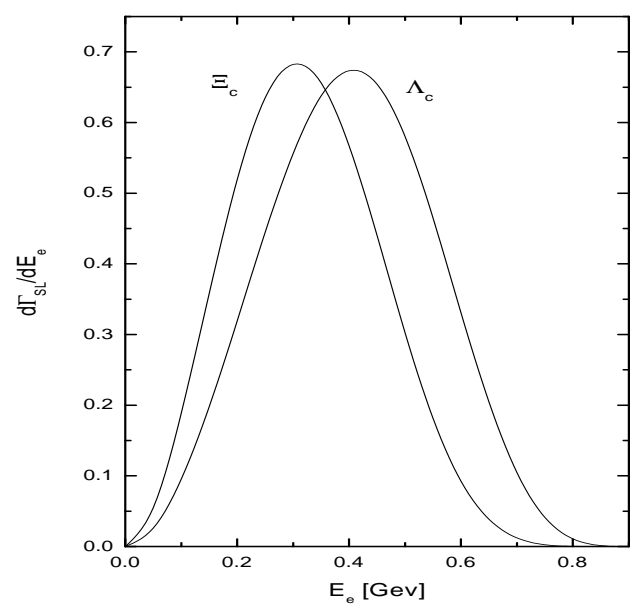

Figure 3. The predicted electron energy spectrum for $\Lambda_{c}$ and $\Xi_{c}$ semileptonic decays. The spectra normalized to $\Gamma_{S L}\left(\Lambda_{c}\right)=0.267 \mathrm{ps}^{-1}$ and $\Gamma_{S L}\left(\Xi_{c}\right)=0.236$ $\mathrm{ps}^{-1}$

The $\Xi_{b}$ inclusive semileptonic rate in the Kalman-D'Souza model is found to be $\Gamma\left(\Xi_{b} \rightarrow\right.$ $\left.X_{c} e \nu_{e}\right)=0.092\left|V_{c b} / 0.04\right|^{2} \mathrm{ps}^{-1}$. Note that $\Gamma_{S L}\left(\Lambda_{b}\right)$ and $\Gamma_{S L}\left(\Xi_{b}\right)$ show $\sim 5 \%$ flavour dependence due to $i$ ) the difference of the $u$ and s constituent quark masses and $i i)$ the difference of $M_{\Lambda_{b}}$ and $M_{\Xi_{b}}$. Both quantities modify $\left|\psi\left(x, p_{\perp}^{2}\right)\right|^{2}$ marginally.

For the charm decays our result are $\Gamma\left(\Lambda_{c} \rightarrow\right.$ $\left.X_{s} e \nu_{e}\right)=0.267 \mathrm{ps}^{-1}$, and $\Gamma\left(\Xi_{c} \rightarrow X_{s} e \nu_{e}\right)=$ $0.236 \mathrm{ps}^{-1}$. The prediction for $\Gamma\left(\Lambda_{c} \rightarrow X_{s} e^{+} \nu\right)$ compares favorably with the estimation based on the branching ratio $\mathcal{B R}\left(\Lambda_{c} \rightarrow e^{+}+\right.$anything $)=$ $(4.5 \pm 1.7) \%$ and the $\Lambda_{c}$ lifetime $\tau_{\Lambda_{c}}=0.208$ ps: $\Gamma\left(\Lambda_{c} \rightarrow X_{s} e^{+} \nu\right)=0.206 \pm 0.07 \mathrm{ps}^{-1}$.

Using the experimental value $\Gamma\left(\Lambda_{c} \rightarrow \Lambda_{s} e^{+} \nu\right)$ measured by CLEO Collaboration one obtains

$R_{E}\left(\Lambda_{c}\right)=\frac{\Gamma\left(\Lambda_{c} \rightarrow \Lambda_{s} e^{+} \nu\right)}{\Gamma\left(\Lambda_{c} \rightarrow X_{c} e^{+} \nu\right)}=(35 \pm 8) \%$.

\section{Conclusions}

This is the most consistent quark model todate describing baryons in the sense that a single set of parameters is used for the whole spectra and then subjecting all of their properties namely the masses, radii and their wavefunction structures to a strict test by a decay model. The spectroscopy is success- fully calculated using far less parameters than any other model [7]. Decays of baryons are calculated. Now in this paper we have successfully computed the electron spectra in the inclusive semileptonic decays of the heavy baryons $H_{Q}$ by incorporating the LF formalism. The existing experimental data are very scarce. There are only a few upper limits on the total semileptonic widths of heavy baryons. The further experimental investigation of the semileptonic decays of heavy flavor baryons will shed the light upon the validity of $\mathrm{OPE}$ in the baryon sector and allows us to test existing quark models of heavy baryons.

This work was supported in part RFBR grants Refs.00-02-16363 and 00-15-96786.

\section{REFERENCES}

1. I.Bigi et al., Ann. Rev. Nucl. Part. Sci, 47 (1997) 591

2. G.Altarelli et al., Nucl. Phys. B202 (1982) 512

3. N. Isgur et al., Phys. Rev. D39 (1989) 799.

4. C.H.Jin, M.F.Palmer, and E.A.Paschos, Phys. Lett. B329 (1994) 364

5. V.L.Morgunov, K.A.Ter-Martirosyan, Phys. Atom. Nucl. 59 (1996) 1221; I.L.Grach et al., Nucl. Phys. B592 (1997) 227

6. S.Kotkovsky et al., Phys. Rev. D60(1999) 114024

7. C.S.Kalman and B.Tran, Nuovo Cimento $\mathbf{1 0 4}$ (1991) 177, C.Kalman and I.D'Souza, Nucl. Phys. B (Proc. Suppl.) 75B (1999) 3

8. B.Block et al., Phys. Rev. D49 (1994) 3356

9. N.B.Demchuck et al., Phys. At. Nucl. 59 (1996) 2152

10. I.Bigi et al., Phys. Lett. B328 (1994) 431

11. I.L.Grach, P.Yu.Kulikov, and I.M.Narodetskii, JETP Letters 73 (2001) 317

12. CLEO Collaboration, B. Barish et al., Phys. Rev. Lett. 76 (1996) 1570.

13. M. Jezabek and J.H. Kühn, Nucl. Phys. B320 (1989) 20.

14. PDG00: Euro. Phys. J C15 (2000) 1

15. I.D'Souza et al., Nucl. Phys. Proc. Suppl. 93 (2001) 3

16. M.Artuso and E.Barberio, arXiv:hep-ph/0205163

17. J.G.Koerner, B.Meloc, hep-ph/0010188 